\title{
On the Singular Points of Plane Curves.
}

By T. B. Sprague, M.A., LL.D.

In the general equation of a curve that passes through the origin, $a_{1} x+b_{1} y+a_{2} x^{2}+b_{z} x y+c_{2} y^{2}+a_{3} x^{3}+b_{3} x^{2} y+c_{3} x y^{2}+d_{3} y^{3}+\ldots=0$, which we may write $u_{1}+u_{2}+u_{3}+u_{4}+\ldots=0$, .

$u_{1} \equiv a_{1} x+b_{1} y=0$ is the equation to the tangent at the origin; for, when $x$ and $y$ are very small, we may neglect all the terms in comparison with those involving the first powers of $x$ and $y$. If neither $a_{1}$ nor $b_{1}$ vanishes, we may, without loss of generality, write the equation in the form

$$
y=m x+u_{2}+u_{3}+u_{4}+
$$

Suppose that, when $y$ is put equal to $m x$, the right hand member becomes $m x+\mathrm{U}_{2} x^{2}+\mathrm{U}_{3} x^{3}+\mathrm{U}_{4} x^{4}+\ldots \ldots$. Then, putting $y=m x$ in the terms of two dimensions in (3), we have approximately $y=m x+\mathrm{U}_{2} x^{2}$.

By means of this equation we find the radius of curvature at the origin to be

$$
\mathrm{R}_{0}=\frac{\left(1+m^{2}\right)^{\frac{3}{2}}}{2 \mathrm{U}_{2}}
$$

The curve will, in the neighbourhood of the origin, lie wholly above or wholly below the tangent at the origin, according as $U_{a}$ is positive or negative. If this quantity vanishes, $\mathbf{R}_{0}$ is infinite. In this case $y-m x$ is a factor of $u_{z}$. Putting $y=m x$ in equation (3), and neglecting terms that involve higher powers of $x$ than the third, we have now $y=m x+\mathrm{U}_{3} x^{3}$. Here the term involving $x^{3}$ changes sign as $x$ passes through the value zero; and the curve, therefore, no longer lies on the same side of the tangent on both sides of the origin, but there is a point of inflection. The tangent at the origin meets the curve in three coincident points. In this case the normal at the origin is an asymptote to the evolute. (See Fig. 8.) ${ }^{*}$ If we draw a line through the origin, cutting the curve in two neighbour-

* In all the figures the darker lines represent the evolute. 
ing points, and the line then moves so as to become the tangent at the origin, those two points move up and coincide with the origin, so that the three coincident points are fully accounted for.

If it should happen, however, that $y-m x$ is a factor also of $u_{3}$, we have $U_{3}=0$; the term involving $x^{3}$ vanishes, and we have to take the next higher term. Reasoning as above, we now get the approximate equation to the curve, $y=m x+\mathrm{U}_{4} x^{4}$. In this case, since $U_{4} x^{4}$ does not change sign as $x$ passes through zero, the curve lies wholly on one side of the tangent in the neighbourhood of the origin; and there is no point of inflection, but we have a point of "undulation". The radius of curvature is still infinite, since $U_{2}$ is still zero, and the normal at the origin is still an asymptote to the evolute; but now both branches of the evolute touch the normal at infinity on the same side of the tangent; in fact, there is a cusp at infinity on the evolute. (See Fig. 9.) The tangent at the origin meets the curve in four coincident points; but it is not possible to explain these as in the former case.

If further terms in the expansion of $y$ vanish, and the first that does not vanish when $m x$ is put for $y$, is the one involving $x^{r}$, then there will be a point of inflection or not at the origin, according as $r$ is odd or even. The tangent at the origin will meet the curve in $r$ coincident points; but any other line through the origin, will meet it there in only one point.

It may now be useful to consider the case where $R_{0}$ is a maximum or minimum. If $y=m x+\mathrm{A} x^{2}+\mathrm{B} x^{3}+\mathrm{C} x^{4}+\ldots$, we find that

$$
\mathbf{R}=\mathbf{R}_{0}\left\{1+\left(\frac{6 m A}{1+m^{2}}-\frac{3 B}{A}\right) x+\text { terms involving } x^{2}, x^{3} \text {, etc. }\right\}
$$

When $x$ is small, $\mathbf{R}-\mathbf{R}_{0}$ changes sign with $x$; but if the coefficient of $x$ in the above value of $R$, vanishes, and the coefficient of $x^{2}$ remains finite, $R-R_{0}$ will no longer change sign, and $R_{0}$ will be a maximum or minimum value of $R$. In consequence of this, there will be a cusp in the evolute at the point corresponding to the origin. (See Fig. 10.) The equation (4) shows that the condition for this is

$$
\mathrm{B}=\frac{2 m \mathrm{~A}^{2}}{1+m^{2}} \text {. }
$$

The origin is in this case a special kind of point; but, as its properties are generally lost on projection, it is not reckoned among the singular points. I propuse to call such a point an "apse". 
Kegarding only the real portions of the curves, the parobola has one apse, and its evolute has one cusp; the hyperbola has two apses, and its evolute has two cusps; and the ellipse has four apses, and its evolute four cusps. If $m=0$, we must have $B=0$ when there is an apse, and the equation becomes $y=\mathrm{A} x^{2}+\mathrm{C} x^{4}+\ldots$.

If both $a_{1}$ and $b_{1}$ vanish, so that the equation reduces to

$$
u_{2}+u_{3}+u_{4}+\ldots=0 \text {, }
$$

the form of the curve in the neighbourhood of the origin is determined by equating to zero the terms of lowest degree; thus, $u_{2}=0$; and this shows that any line through the origin meets the curve there in two coincident points. We then get two values of $y / x$, which may be real and unequal, or equal, or imaginary. First, suppose that the values are real and unequal, namely, $m$ and $p$; then we may, without loss of generality, take the equation to the curve to be $\quad(y-m x)(y-p x)=u_{3}+u_{\downarrow}+\ldots$.

This indicates that there are two tangents at the origin, $y-m x=0$, and $y-p x=0$, each of which meets the curve in three coincident points there ; in other words, the origin is a double point or "node". Taking the former of the two tangents, and putting $y=m x$ in $\frac{u_{3}}{y-p x}$, we get approximately $y=m x+\frac{\mathrm{U}_{3}}{m-p} x^{2}$.

The radius of curvature of this branch at the origin is $\frac{m-p}{2 \mathrm{U}_{3}}\left(1+m^{2}\right)^{\frac{3}{3}}$. If $m-p=0$ and $\mathrm{U}_{3}$ is finite, which is a case we shall consider presently, the radius of curvature is zero. If, on the other hand, $\mathrm{U}_{3}=0$, and $m-p$ not $=0$, then the radius of curvature is infinite. In this case, $y-m x$ is a factor of $u_{3}$, and the equation to the curve becomes $(y-m x)(y-p x)=(y-m x) v_{:}+u_{4}+\ldots$,

whence

$$
y=m x+\frac{u_{4}+\ldots \ldots}{y-\mu x-v_{2}},
$$

and approximately $\quad y=m x+\frac{\mathrm{U}_{4}}{m-p} x^{3}$.

This shows that the branch has a point of inflection at the origin; and the tangent $y-m x=0$ will therefore meet the origin in three coincident points; but it also cuts the other branch, and therefore 
meets the curve in four coincident points at the origin. If $y-p x$ also is a factor of $u_{3}$, then the second branch also has a point of inflection at the origin.

Figure 11 represents a case where one branch has a point of inflection at the origin, and the other has not; and fig. 12 represents a case where each branch has such a point. Salmon calls these points "flecnode" and "biflecnode", respectively.

If $y-m x$ is also a factor of $u_{4}$, the origin will be a point of undulation in the branch ; and if $y-p x$ also is a factor of $u_{4}$, there will be an undulation in each branch. The effect of these singularities on the evolute is evident from what was said above.

Next suppose that the roots of $u_{2}=0$ are imaginary, so that there are two imaginary tangents at the origin. The equation to the curve cannot then be satisfied by any very small real values of $x$ and $y$ except $x=0, y=0$; there are therefore no real points adjacent to the origin, which is thus an isolated point on the curve, and is called a "conjugate" point, or, by Salmon an "acnode". The former of these terms is objectionable, because it puts out of sight the fact that the point must always, like a node, count as two points. I propose to call such a point a "doublet". Any real line through the origin meets the curve in two coincident real points there; but each of the imaginary tangents meets the curve in three coincident real points at the origin.

Lastly, suppose that the roots of $u_{2}=0$ are equal, so that $m=p$; then we may take the equation of the curve to be $(y-m x)^{2}=u_{3}+u_{4}+\ldots \ldots$; and putting $y=m x$ in $u_{3}$, we get approximately $(y-m x)^{2}=\mathrm{U}_{9} x^{2}$, and

$$
y=m x \pm \sqrt{ } \mathrm{U}_{3} \cdot x^{\frac{3}{2}}
$$

If $U_{3}$ is positive, $x$ cannot be negative; and the equation shows that the curve has two branches, each of which touches $y-m x=0$ at the origin. The origin is therefore a cusp: (see Fig. 13). The value of $\frac{d^{2} y}{d x^{2}}$ at the origin is infinite, unless $\mathrm{U}_{3}=0$; and the radius of curvature is therefore zero, and the evolute of the curve passes through the origin. The tangent at the origin still meets the curve in three coincident points. If $\mathrm{U}_{3}$ is negative, $x$ cannot be positive; but there is still a cusp at the origin, with its angle pointed in the opposite direction. 
If it should happen that $U_{3}=0$, so that $y-m x$ is a factor of $u_{3}$, the equation to the curve takes the form

$$
(y-m x)^{2}=(y-m x) v_{2}+u_{4}+\ldots
$$

whence

$$
\left(y-m x-\frac{1}{2} v_{3}\right)^{2}=\frac{1}{4} v_{3}^{2}+u_{4}+\ldots
$$

and

$$
y=m x+\frac{1}{2} v_{2} \pm \sqrt{\frac{1}{4} v_{2}^{2}+u_{4}+\ldots}
$$

or approximately $y=m x+\frac{1}{2} \mathrm{~V}_{2} x^{2} \pm \sqrt{\frac{1}{4} \mathrm{~V}_{2}^{2}+\mathrm{U}_{4}} \cdot x^{2}$

$$
=m x+\left(\frac{1}{2} \mathrm{~V}_{2} \pm \sqrt{\left.\frac{1}{4} \mathrm{~V}_{2}{ }^{2}+\mathrm{U}_{4}\right)} x^{2}\right.
$$

where $\mathrm{V}_{2}$ is the value of $v_{2} / x^{2}$ when $y$ is put equal to $m x$. In this case the curve has two branches, which are of finite and different eurvatures; and both touch the line $y-m x=0$ at the origin. This singularity has been called a tacnode. In this case the normal at the origin is a bitangent to the evolute : (see Fig. 14). The tangent at the origin meets the curve in four coincident points; and any other line through the origin meets it in two coincident points. If we take a line, parallel to the tangent, and cutting the curve in four points, and this line moves parallel to itself and becomes the tangent at the origin, then all these four points coincide with the origin, and the four coincident points are thus fully accounted for. In some other cases the full number of coincident points can be similarly accounted for, by means of the real branches of the curve; for instance, in the cases of the inflection, as we have already seen, in the node, and the cusp.

There may be an apse in either or both of the branches. For the sake of simplicity, we will now put $m=0$, so that the equation becomes

$$
y=\frac{1}{2} v_{2} \pm \sqrt{\frac{1}{4} v_{2}^{2}+u_{4}+u_{5}+\ldots} \text {. }
$$

Suppose that

$$
v_{2}=\mathrm{A} x^{2}+\mathrm{B} x y+\mathrm{C} y^{2} \text {. }
$$

Then, putting $y=0$ in the right-hand member of (7) we get as a first approximation

$$
\begin{aligned}
y & =! \mathrm{A} x^{2} \pm \sqrt{\frac{1}{4} \mathrm{~A}^{2} x^{d}+a_{4} x^{4}} \\
& =\left(\frac{1}{2} \mathrm{~A} \pm \sqrt{\frac{1}{4} \mathrm{~A}^{2}+a_{4}}\right) x^{2} \\
& =\mathrm{H} x^{2}, \text { suppose. }
\end{aligned}
$$


Then, giving $y$ this value in the equation (7), we have for a second approxination

$$
\begin{aligned}
y & =\frac{1}{2} \mathrm{~A} x^{2}+\frac{1}{2} \mathrm{BH} x^{3} \pm \sqrt{\frac{1 \mathrm{~A}^{2} x^{4}+\frac{1}{2} \mathrm{ABH} x^{5}+a_{6} x^{4}+b_{4} \mathrm{H} x^{5}+a_{5} x^{5}}{2}} \\
& =\frac{1}{2} \mathrm{~A} x^{2}+\frac{1}{2} \mathrm{BH} x^{3} \pm \sqrt{\left(\frac{1}{4} \mathrm{~A}^{2}+a_{4}\right) x^{4}+\left(\frac{1}{2} \mathrm{AB} \overline{\mathrm{H}}+b_{4} \mathrm{H}+a_{5}\right) x^{5}} \\
& =\mathrm{H} x^{2}+\left(\frac{1}{2} \mathrm{BH} \pm \frac{\frac{1}{2} \mathrm{ABH}+b_{4} \mathrm{H}+a_{8}}{2 \sqrt{\frac{1}{4} \mathrm{~A}^{2}+a_{4}}}\right) x^{3} .
\end{aligned}
$$

In order that there may be an apse, the co-fficient of $x^{s}$ must vanish, or $\mathrm{BH} \sqrt{\overline{1} \mathrm{~A}^{2}+a_{4}} \pm\left(\frac{1}{2} \mathrm{ABH}+b_{4} \mathrm{H}+a_{5}\right)=0$;

which, on substituting for $H$ its value, becomes

$$
\pm\left(\mathrm{AB}+b_{4}\right) \sqrt{\frac{1}{4} \mathrm{~A}^{2}+a_{4}}+\frac{1}{2} \mathrm{~A}^{2} \mathrm{~B}+a_{4} \mathrm{~B}+\frac{1}{2} \mathrm{~A} b_{4}+a_{5}=0 .
$$

The one or the other of the branches has an apse, according as we take the upper or lower sign. But if

$$
\mathrm{AB}+b_{4}=0 ; \text { and } \frac{1}{2} \mathrm{~A}^{2} \mathrm{~B}+a_{4} \mathrm{~B}+\frac{1}{2} \mathrm{~A} b_{4}+a_{5}=0 \text {, }
$$

the co-efficient of $x^{3}$ will vanish, whichever sign is taken, and there is an apse on each branch. The above equations are equivalent to

$$
b_{4}=-\mathrm{AB}, a_{5}=-a_{4} \mathrm{~B} ;
$$

and the equation to the curve becomes

$$
\begin{aligned}
y^{2}-y\left(\mathrm{~A} x^{2}+\mathrm{B} x y+\mathrm{C} y^{2}\right) & +a_{4} x^{4}-\mathrm{AB} x^{3} y+c_{8} x^{2} y^{2} \\
& +d_{4} x y^{3}+e_{4} y^{4}-a_{4} \mathrm{~B} x^{5}+b_{5} x^{4} y+\ldots=0 .
\end{aligned}
$$

If there is one apse, the evolute has one cusp, as shown in Fig. 15; and if there are two apses, the evolute has two cusps, as shown in Fig. 16.

If $U_{4}$ is negative and $\left|U_{4}\right|>\frac{1}{4} \nabla_{2}^{2}$, the value of $y$ given by equation (6) is imaginary except when $x=0, y=0$; any line through the origin meets the curve there in two coincident points, and the line $y-m x=0$ meets the curve there in four coincident points :-just as in the case of a tacnode. The curre has thus an isolated point at the origin, but it is not a doublet, and there is a real bitangent at the origin, $y-m x=0$; or, rather, two coincident tangents, which both touch the imaginary branch at the origin.

We have a particular case of this when $u_{3}$ is absent from the equation in consequence of its coefficients vanishing, and $u_{t}$ is a negative square, so that the equation takes the form

$$
(y-m x)^{2}+\left(p x^{2}+q x y+r y^{2}\right)^{2}=u_{5}+\ldots .
$$


If $U_{4}=0$, so that $y-m x$ is a factor of $u_{4}$, and $u_{4}=(y-m x) v_{3}$ we have $\quad\left(y-m x-\frac{1}{2} v_{2}-\frac{1}{2} v_{3}\right)^{2}=\frac{1}{4}\left(v_{2}+v_{3}\right)^{2}+u_{B}+\ldots$

and

$$
\begin{aligned}
y & =m x+\frac{1}{2} v_{2}+\frac{1}{2} v_{3} \pm \frac{1}{2}\left(v_{2}+v_{3}\right) \sqrt{1+\frac{4 u_{5}}{\left(v_{2}+v_{3}\right)^{2}}+\ldots} \\
& =m x+\frac{1}{2}\left(v_{2}+v_{3}\right) \pm \frac{1}{2}\left\{v_{3}+v_{3}+\frac{2 u_{5}}{v_{2}+v_{3}}+\ldots\right\} .
\end{aligned}
$$

Taking the upper sign, we get

$$
y=m x+v_{2}+v_{3}+\frac{u_{5}}{v_{2}+v_{3}}+\ldots=m x+\mathrm{V}_{2} x^{2} \text { approximately. }
$$

But, taking the lower sign, we have

$$
y=m x-\frac{u_{5}}{v_{2}+v_{3}}-\ldots=m x-\frac{\mathrm{U}_{5}}{\mathrm{~V}_{2}} x^{3}, \quad \text { approximately. }
$$

This shows that one branch has an inflection at the origin, which may be therefore called a "tacflecnode". (See Fig. 17.) In this case the tangent at the origin meets the curve in five coincident points. The normal touches one branch of the evolute, and is an asymptote to another branch.

If $\mathrm{V}_{2}=0$, so that $v_{2}=(y-m x) v_{1}$, we have

$$
(y-m x)^{2}=(y-m x)^{2} v_{1}+u_{4}+u_{5}+\ldots
$$

and we may neglect $(y-m x)^{2} v_{1}$ in comparison with $(y-m x)^{2}$, so that

$$
y=m x \pm \sqrt{ } \mathrm{U}_{4} \cdot x^{2} \text { approximately. }
$$

In this case the twio branches have the same curvature, but are turned in opposite directions, as in Fig. 18.

If $U_{4}$ and $\mathrm{V}_{2}$ both $=0$, so that $u_{4}=(y-m x) v_{3}$, we have

$$
(y-m x)^{2}=(y-m x)^{2} v_{1}+(y-m x) v_{3}+u_{5}+\ldots .
$$

In this case the method we have followed hitherto is not applicable. In order to get an approximate equation to the curve, we must neglect those terms which are of a higher degree than the others: thus, as already mentioned, we may neglect $(y-m x)^{2} v_{1}$ in comparison with $(y-m x)^{2}$; but we cannot say which of the remaining terms above written down is of highest degree, and may be neglected, until we know the degree of $(y-m x)$. We may determine this by trial. Assume that $(y-m x)^{2}$ and $(y-m x) v_{3}$ are of the same degree ; 
then $y-m x$ must be of the third degree, and each of the two terms is of the sixth degree : but $u_{5}$ is only of the fifth degree, and therefore must not be neglected. Our assumption, that $(y-m x)^{2}$ and $(y-m x) v_{3}$ are of the same degree, is therefore inadmissible. Next assume that $(y-m x)^{2}$ and $u_{8}$ are of the same degree; then $y-m x$ is of the degree $2 \frac{1}{2}$, and each of these terms is of the degree $5:$ then $(y-m x) v_{3}$ is of the degree $5 \frac{1}{2}$, and may be neglected in comparison with the other two. Lastly, assume that $(y-m x) v_{3}$ and $u_{5}$ are of the same degree; then $y-m x$ is of the second degree; but this makes $(y-m x)^{2}$ of the fourth degree, which is lower than the other two, and this assumption is therefore inadmissible.

The only assumption therefore that is admissible is that $(y-m x)^{2}$ and $u_{5}$ are of the same degree ; and putting $y=m x$ in the latter, we get

$$
y-m x= \pm \sqrt{ } \mathrm{U}_{5} \cdot x^{\frac{5}{2}}
$$

In this case there is a cusp at the origin; but the radius of currature is infinite, and I propose to call such a point a "flat cusp". The normal at the origin is an asymptote to the evolute; and as the infinite branches lie on the same side of the normal, there is an inflection at infinity in the evolute. (See Fig. 19.)

If $\frac{1}{4} \nabla_{2}^{2}+U_{4}=0$, but $V_{2}, U_{4}$, are not separately $=0$, the approximate equation (6) becomes $y=m x+\frac{1}{2} \mathrm{~V}_{2} x^{2}$, and both branches have the same curvature at the origin. In order to distinguish between them, we must carry our approximation further. Resuming equation

(5) we have

$$
y=m x+\frac{1}{2} v_{2} \pm \sqrt{\frac{1}{4} v_{2}^{2}+u_{4}+u_{5}+\ldots}
$$

and putting

$$
y=m x+\frac{1}{2} \mathrm{~V}_{2} x^{2} \text { in } v_{2}, u_{4}, u_{3},
$$

we get a result of the form

$$
y=m x+\frac{1}{2} \mathrm{~V}_{\mathrm{g}} x^{2}+\mathrm{M} x^{3}+\mathrm{N} x^{4} \pm \sqrt{\mathrm{P} x^{5}+\mathrm{Q} x^{8}+\mathrm{S} x^{7}+\mathrm{T} x^{8}} .
$$

If $P$ is finite and positive, the approximate equation to the curve is

$$
y=m x+\frac{1}{2} \mathrm{~V}_{2} x^{2} \pm \sqrt{ } \mathbf{P} \cdot x^{\frac{5}{2}}
$$

In this case there is a cusp with both branches on the same side of the tangent. This has been called a ramphoid cusp, as it somewhat resembles the beak of a bird; and I venture to suggest that it should be called a "beak". Salmon calls it a node-cusp.

By applying the formula for $R$, we find that, as already men- 
tioned, the two branches have the same finite radius of curvature at the origin; also that the curvature of one branch increases, and that of the other diminishes, as we recede from the origin.

For, putting $y^{\prime}$ for $\frac{d y}{d x}$ and $y^{\prime \prime}$ for $\frac{d^{2} y}{d x^{2}}$,

and

$$
\begin{gathered}
y^{\prime}=m+\mathrm{V}_{2} x \pm \frac{5}{2} \sqrt{ } \mathbf{P} \cdot x^{\frac{3}{2}}+\ldots \\
y^{\prime \prime}=\mathrm{V}_{2} \pm \frac{15}{4} \sqrt{ } \mathbf{P} x^{\frac{1}{2}}+\ldots \\
\left(1+y^{\prime 2}\right)^{\frac{3}{2}}=\left\{1+m^{2}+2 m \nabla_{2} x \pm 5 m \sqrt{ } \mathbf{P} \cdot x^{\frac{3}{2}}+\ldots\right\}^{\frac{3}{2}} \\
=\left(1+m^{2}\right)^{\frac{3}{2}}\left\{1+\frac{3 m V_{2}}{1+m^{2}} x+\ldots\right\} \\
\mathbf{R}=\frac{\left(1+y^{\prime 2}\right)^{\frac{3}{2}}}{y^{\prime \prime}}=\frac{\left\{1+m^{2}\right\}^{\frac{3}{2}}}{\nabla_{2}}\left\{1 \mp \frac{15}{4} \frac{\sqrt{ } \mathrm{P}}{V_{2}} x^{\frac{1}{2}}+\frac{3 m V_{2}}{1+m^{2}} x\right\} \\
+ \text { termg involving } x^{\frac{3}{2}}, x^{2}, \text { etc. } \\
=\mathbf{R}_{0}\left(1 \mp \frac{15}{4} \frac{\sqrt{ } \mathbf{P}}{V_{2}} x^{\frac{1}{2}}\right) \quad \text { approximately }
\end{gathered}
$$

where we retain only the lowest power of $x$, as all the others may be neglected when $x$ is very small. Bearing in mind that the term $\sqrt{ } \mathbf{P} \cdot x^{\frac{5}{2}}$ in the value of $y$, has a double sign, and denoting by $\mathbf{R}_{1}$, $R_{2}$, the radii of curvature in the two branches near the origin, we have approximately

$$
R_{1}=R_{0}\left(1-\frac{15}{4} \frac{\sqrt{P}}{\nabla_{2}} x^{\frac{1}{3}}\right) ; \quad R_{2}=R_{0}\left(1+\frac{18}{1} \frac{\sqrt{ } \mathrm{P}}{V_{2}} x^{\frac{1}{2}}\right)
$$

There is thus an inflection in the evolute at the point corresponding to the origin. The form of the curve at the origin and of the evolute at the corresponding point, are shown in Fig. 20.

There is, in general, as Salmon points out, no point of inflection in the evolute. ('The same is the case in any envelop, and the evolute is a particular kind of envelop). The above is, therefore, one of the exceptional cases in which the evolute has an inflection. I have hitherto been unable to identify this exceptional case with one of those indicated by Salmon; but I hesitate to suggest that there has been any oversight on his part, as I have found the whole of his reasoning as to the singular points of the evolute, extremely difficult to follow. 
If $\mathbf{P}=0$, the approximate equation to the curve takes the form

$$
y=m x+\frac{1}{2} \mathrm{~V}_{2} x^{2}+(\mathrm{M} \pm \sqrt{ } \mathrm{Q}) x^{3}
$$

and there is now no cusp, but a tacnode at the origin; and as both branches of the curve have the same radius of curvature at the origin, there is a tacnode in the evolute. (See Fig. 21).

If

$$
\mathrm{M}+\sqrt{ } \mathrm{Q} \text { or } \mathrm{M}-\sqrt{ } \mathrm{Q}=\frac{m}{1+m^{2}} \cdot \frac{\mathrm{V}_{2}^{2}}{2}
$$

there is an apse in one branch of the curve, and a cusp in the evolute. (See Fig. 22).

But if $Q$ also $=0$, the equation takes the form

$$
y=m x+\frac{1}{2} \nabla_{q} x^{2}+M x^{3} \pm \sqrt{ } \mathrm{S} \cdot x^{\frac{2}{2}}
$$

In this case there is again a ramphoid cusp or "beak"; but it differs from the one considered above, inasmuch as the radius of curvature is now a maximum or minimum at the cusp. For we now have

$$
\mathbf{R}=\mathbf{R}_{0}\left\{1+\left(\frac{3 m \mathrm{~V}_{2}}{1+m^{2}}-\frac{6 \mathrm{M}}{\mathrm{V}_{2}}\right) x \pm \frac{35}{4} \frac{\sqrt{ } \mathrm{S}}{\mathrm{V}_{2}} x^{\frac{3}{2}}\right\}
$$

and this shows that, unless $M=\frac{m V_{2}^{2}}{2\left(1+m^{2}\right)}$, so that the co-efficient of $x$ vanishes, $R_{1}$ and $R_{2}$ are either both greater or both less than $R$. In this case there is a "beak" in the evolute, as shown in Fig. 23.

If $M$ has this value, $R$ is no longer a maximum or minimum at the cusp, and there is now again an inflection in the evolute, as in Fig. 20.

If $a_{2}, b_{2}, c_{2}$ all vanish, as well as $a_{1}, b_{1}$, the equation becomes

$$
u_{3}+u_{4}+u_{5}+\ldots=0 \text {. }
$$

Equating $u_{3}$ to zero, we have three values of $y / x$, or the origin is a triple point. The three values may be (1) all real and unequal, or (2) two of them may be equal, or (3) two of them may be imaginary, or (4) all three may be equal. It is unnecessary to investigate cases (1), (2), or (3), as the branches may have the singularities we have investigated above, and no others. The only case that remains is that in which the three values of $y / x$ are equal. We may then take the equation to be

$$
(y-m x)^{3}=u_{4}+u_{3}+\ldots
$$


Proceeding in the usual way, we get the approximate equation to the curve

$$
y=m x+\sqrt[3]{\mathrm{U}_{4}} \cdot x^{\frac{4}{3}}
$$

There is only one real branch to the curve, and the tangent at the origin meets the curve in four coincident points; while any other line through the origin meets the curve in three coincident points. There is not, however, a doublet at the origin; for the tangent at a doublet is imaginary, the equation in that case being of the form

$$
(y-m x)\left\{(a x+b y)^{2}+c^{2} y^{2}\right\}+u_{4}+u_{5}+\ldots=0 .
$$

The radius of curvature at the origin vanishes, and the evolute passes through the origin, and has a cusp there. (See Fig. 24.) The origin would be a point of "undulation" if the radius of curvature were infinite; perhaps we may call the present singularity a point of "condensation".

If $U_{1}=0$, so that $y-m x$ is a factor of $u_{4}$, then the equation takes the form $\quad(y-m x)^{3}=(y-m x) v_{3}+u_{5}+u_{6}+\ldots$

and we have to determine by trial which of these terms may be neglected in comparison with the others.

Since $y=m x$ is the tangent at the origin, $x$ and $y$ are of the same degree, and we may neglect $u_{6}$ in comparison with $u_{5}$, if the latter does not contain the factor $y-m x$. The equation therefore becomes approximately

$$
(y-m x)^{3}=(y-m x) v_{3}+u_{5} .
$$

If now we assume provisionally that $u_{5}$ may be neglected in comparison with the other terms, we get $(y-m x)^{2}=v_{3}$; and putting $m x$ for $y$ in $v_{3}$ we get $y=m x \pm \sqrt{ } V_{3}, x^{3}$. The terms we have kept are of the degree $4 \frac{1}{2}$, and it is therefore correct to reject $u_{5}$ in comparison with them.

Next assume that $(y-m x) v_{3}$ may be neglected. This gives $(y-m x)^{3}=\mathrm{U}_{5} x^{5}$. The terms retained are now of the degree 5 , but the one neglected is of the degree $4 \frac{2}{3}$, and our assumption is therefore inadmissible. 
Lastly, assume that $(y-m x)^{3}$ may be neglected; then we have $y-m x=-\frac{u_{5}}{v_{3}} ;$ and when we put $y=m x$ on the right hand side, $y=m x-\frac{U_{5}}{V_{3}} x^{2}$. This makes the degree of the terms kept, 5 , while the degree of the other is 6 , and it is therefore rightly neglected.

It does not seem worth while to give the working out of other cases, and I will therefore only give some results. For brevity put $y-m x=Z$. We have seen that when $U_{4}=0$, or the equation takes the form

$$
Z^{3}=Z v_{3}+u_{5}+u_{6}+\ldots,
$$

the curve has three real branches, for which we have approxinately at the origin

$$
\mathrm{Z}= \pm \sqrt{ } / \mathrm{V}_{3} \cdot x^{\frac{3}{2}} \text { or }-\frac{\mathrm{U}_{5}}{V_{3}} \cdot x^{2}
$$

If now $V_{3}=0$, the equation becomes

$$
Z^{3}=Z \cdot v_{2}+u_{5}+u_{6}+\ldots
$$

and there is only one real branch to the curve, for which $Z=\sqrt[3]{U_{5}} . x^{3.5}$. In this case there is a point of inflection at the origin; but it is not an ordinary inflection, as the radius of curvature is not infinite, but vanishes. I would propose to call such a point a "twist". In this case the evolute passes through the origin, and has an inflection there. (See Fig. 26.)

If $\mathrm{U}_{5}=0$, but $\mathrm{V}_{3} \neq 0$, we have

$$
\mathrm{Z}^{3}=\mathrm{Z}\left(v_{3}+v_{4}\right)+u_{6}+\ldots
$$

and

$$
\mathrm{Z}= \pm \sqrt{\mathrm{V}_{3}} \cdot x^{\frac{3}{2}} \text { or }-\frac{\mathrm{U}_{6}}{\mathrm{~V}_{3}} \cdot x^{3}
$$

If $V_{3}$ and $U_{5}$ both $=0$, the equation becomes

$$
\mathrm{Z}^{3}=\mathrm{Z}^{2} v_{2}+\mathrm{Z} v_{4}+u_{6}+\ldots
$$

and if the roots of $Z^{3}-V_{2} Z^{2}-V_{4} Z-U_{6}=0$

are $z_{1}, z_{2}, z_{3}$, and real, the curve has three branches for which

$$
\mathrm{Z}=z_{1} x^{2}, z_{2} x^{2}, z_{3} x^{2} \text {, respectively. }
$$

In this case the normal at the origin will touch three branches of the evolute. (See Fig. 28.) If $z_{1}=z_{2}=z_{3}$, so that all three branches of the curve have the same radius of curvature at the origin, the 
three points of contact of the normal with the evolute, will coincide; and we shall have what may be called a triple tacnode in the evolute (see Fig. 29), which, if the origin should be an apse in each branch of the curve, will become a triple cusp. (See Fig. 30.)

If $\mathrm{U}_{6}=0$, so that one of the roots of the cubic vanishes, we have

$$
Z^{3}=Z^{2} v_{2}+Z\left(v_{4}+v_{5}\right)+u_{5}+\ldots
$$

and

$$
\mathrm{Z}=z_{1} x^{2}, z_{2} x^{2}, \text { or }-\frac{\mathrm{U}_{\overline{7}}}{\mathrm{~V}_{4}} x^{3}
$$

If $\mathrm{U}_{z}$ also $=0$, we have

$$
Z^{3}=Z^{2} v_{2}+Z\left(v_{4}+v_{5}+v_{6}\right)+u_{8}+\ldots
$$

and the third branch becomes

$$
Z=-\frac{U_{8}}{V_{4}} \cdot x^{4} .-\quad-\quad \text { (See Fig. 32.) }
$$

In these last two cases the values of $z_{1}, z_{2}$ are

$$
\left\{V_{2} \pm \sqrt{V_{2}^{2}+4 V_{4}}\right\}
$$

If $\mathrm{V}_{4}=0$, but $\mathrm{U}_{7} \neq 0$, we have

$$
Z^{3}=Z^{2}\left(v_{2}+v_{3}\right)+Z v_{5}+u_{7}+\ldots
$$

and

$$
\mathrm{Z}=\mathrm{V}_{\mathrm{g}} x^{2} \text { or } \pm \sqrt{ }-\frac{\mathrm{C}_{7}}{\mathrm{~V}_{2}} \cdot w^{\frac{5}{2}}
$$

Going back to equation (8), if $z_{1}=z_{2}=z_{3}$, we have

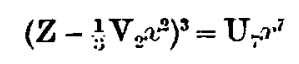

and

$$
\mathbf{Z}=\frac{1}{3} \mathbf{V}_{2} x^{2}+\sqrt[3]{\mathbf{U}_{7}} \cdot x^{\frac{7}{3}}
$$

If $\mathrm{V}_{2}$ and $\mathrm{U}_{6}$ both $=0$, we have

and

$$
\mathrm{Z}^{3}=\mathrm{Z}^{i} v_{1}+\mathrm{Z}\left(v_{\mathrm{1}}+v_{\mathrm{i}}\right)+u_{\mathrm{7}}+\ldots
$$

If both $V_{4}$ and $U_{6}=0$, we have

$$
Z= \pm \sqrt{ } V_{4}, x^{2} \text { or }-\frac{U_{5}}{V_{4}} \cdot x^{3}
$$

$$
Z^{3}=Z^{2}\left(v_{2}+v_{3}\right)+Z v_{3}+u_{7}+\ldots
$$

and

$$
\mathrm{Z}=\mathrm{V}_{\mathrm{2}} \mathrm{x} \text { or } \pm \sqrt{ }-\frac{\mathrm{U}_{7}}{\mathrm{~V}_{2}} \cdot x^{\frac{5}{2}}
$$

If $\mathrm{V}_{3}, \mathrm{~V}_{4}$, and $\mathrm{U}_{6}$, all $=0$, we have

$$
Z^{3}=Z^{3} v_{1}+Z^{2} v_{3}+Z v_{5}+u_{7}+\ldots
$$

and

$$
Z=\sqrt[3]{U_{7}}, x^{\frac{7}{3}}
$$


If here $\mathrm{U}_{7}=0$, we have

$$
\mathrm{Z}^{3}=\mathrm{Z}^{3} v_{1}+\mathrm{Z}^{2} v_{3}+\mathrm{Z}\left(v_{5}+v_{8}\right)+u_{8}+\ldots
$$

and

$$
Z= \pm \sqrt{ } V_{5} \cdot x^{\frac{5}{2}} \text { or }-\frac{U_{8}}{\nabla_{8}^{3}} x^{3} \text { - - (See Fig. 38.) }
$$

If $\mathrm{V}_{5}$ also $=0$, we have

$$
Z^{3}=Z^{y} v_{1}+Z^{2}\left(v_{s}+v_{4}\right)+Z v_{8}+u_{s}+\ldots
$$

and

$$
Z=\because / \mathrm{U}_{8} \cdot x^{3} \quad \cdot \quad-\quad \cdot
$$

Again, supposing the equation to be reduced to

$$
u_{4}+u_{5}+\ldots=0,
$$

the only case which it is necessary to consider, is the one where there are four real branches at the origin, which all have the same tangent; so that the equation may be written

$$
Z^{4} \equiv(y-m x)^{4}=u_{3}+u_{6}+\ldots .
$$

Proceeding in the usual way, we get as a first approximation

$$
\mathrm{Z} \equiv y-m x= \pm \sqrt{\mathrm{U}_{3}}, x^{\frac{5}{4}} \text {. }
$$

This represents a cusp, at which the radius of curvature is zero; so that the evolute passes through the origin, as in Fig. 13.

If $\mathrm{U}_{5}=0$, so that

$$
\mathbf{Z}^{4}=\mathbf{Z} v_{4}+u_{6}+\ldots
$$

we have

$$
Z=3 / V_{4} \cdot x^{\frac{4}{3}} \text { or }-\frac{U_{6}}{V_{4}} \cdot x^{2}
$$

If $\mathrm{V}_{4}=0$, so that

$$
Z^{4}=Z^{2} v_{3}+u_{6}+\ldots
$$

we have $Z^{2}=p x^{3}$ or $q x^{3}$, where $p, q$ are the roots of $z^{2}-\mathrm{V}_{\mathrm{g}} z-\mathrm{U}_{6}=0$, and

$$
Z=\sqrt{ } p \cdot x^{\frac{3}{2}} \text { or } \sqrt{ } q \cdot x^{3} \cdot
$$

The curve now has two cusps at the origin, with the same tangent ; and there is a tacnode in the evolute. 
If $V_{3}=0$, we have

$$
Z^{4}=Z^{3} v_{2}+u_{6}+\ldots
$$

and

$$
Z= \pm \pm / U_{6} \cdot x^{: \ddot{z}}
$$

which represents a common cusp as in Fig. 13.

But if $U_{6}=0$, we have

and

$$
Z^{4}=Z^{2} v_{3}+Z v_{5}+u_{7}+\ldots
$$

where $p, q$ are the roots of $\mathrm{V}_{3} z^{2}+\mathrm{V}_{5} z+\mathrm{U}_{7}=0$.

If both $\nabla_{3}$ and $U_{6}=0$, we have $Z^{4}=Z^{3} v_{2}+Z v_{5}+u_{4}+\ldots$

and

$$
\mathrm{Z}=\sqrt[4]{\mathrm{U}_{7} \cdot a^{\frac{5}{4}}}
$$

If here $\mathrm{U}_{7}=0$, we have

and

$$
Z^{4}=Z^{3} v_{2}+Z\left(v_{5}+v_{6}\right)+u_{8}+\ldots
$$

$$
Z=\sqrt[3]{\mathrm{V}_{5}} \cdot x^{\frac{5}{3}} \text { or }-\frac{\mathrm{C}_{8}}{\mathrm{~V}_{5}} \cdot x^{3} \text {. }
$$

But if $\nabla_{s}$ also $=0$, we have

$$
\mathrm{Z}^{4}=\mathrm{Z}^{3} v_{2}+\mathrm{Z}^{2} v_{4}+\mathrm{Z} v_{6}+u_{\mathrm{y}}+\ldots
$$

and

$$
Z=p x^{2} \text {, or } q x^{2} \text {, or } r x^{2} \text {, or } s x^{2} \text {, }
$$

where $p, q, r, s$ are the roots of $z^{4}-\mathrm{V}_{2} z^{3}-\mathrm{V}_{4} z^{2}-\mathrm{V}_{8} z-\mathrm{U}_{8}=0$.

One other kind of singular point may be mentioned, namely a beak (or ramphoid cusp) at which the radius of curvature is infinitely small. We have such a point at the origin when

$$
y=m x+a x^{\frac{4}{3}}+b x^{\frac{3}{2}}+\ldots \cdot \quad-\quad \text { (See Fig. 45.) }
$$

The singularity in this case is of a much higher order than any we have considered above; for, on clearing the equation of radicals, there are no terms in it of lower degree than the sixth.

In all cases the figures are drawn as if $m$ were $=0$. 